\title{
Delivering Heparin-Binding Insulin-Like Growth Factor 1 with Self-Assembling Peptide Hydrogels
}

\author{
Emily M. Florine, PhD, ${ }^{1}$ Rachel E. Miller, PhD, ${ }^{2}$ Paul H. Liebesny, BS, ${ }^{1}$ Keri A. Mroszczyk, MS, \\ Richard T. Lee, MD, ${ }^{3}$ Parth Patwari, MD, ScD, ${ }^{4}$ and Alan J. Grodzinsky, ScD ${ }^{1}$
}

Heparin-binding insulin-like growth factor 1 (HB-IGF-1) is a fusion protein of IGF-1 with the HB domain of heparin-binding epidermal growth factor-like growth factor. A single dose of HB-IGF-1 has been shown to bind specifically to cartilage and to promote sustained upregulation of proteoglycan synthesis in cartilage explants. Achieving strong integration between native cartilage and tissue-engineered cartilage remains challenging. We hypothesize that if a growth factor delivered by the tissue engineering scaffold could stimulate enhanced matrix synthesis by both the cells within the scaffold and the adjacent native cartilage, integration could be enhanced. In this work, we investigated methods for adsorbing HB-IGF-1 to self-assembling peptide hydrogels to deliver the growth factor to encapsulated chondrocytes and cartilage explants cultured with growth factor-loaded hydrogels. We tested multiple methods for adsorbing HB-IGF-1 in self-assembling peptide hydrogels, including adsorption prior to peptide assembly, following peptide assembly, and with/without heparan sulfate (HS, a potential linker between peptide molecules and HB-IGF-1). We found that HB-IGF-1 and HS were retained in the peptide for all tested conditions. A subset of these conditions was then studied for their ability to stimulate increased matrix production by gel-encapsulated chondrocytes and by chondrocytes within adjacent native cartilage. Adsorbing HB-IGF-1 or IGF-1 prior to peptide assembly was found to stimulate increased sulfated glycosaminoglycan per DNA and hydroxyproline content of chondrocyte-seeded hydrogels compared with basal controls at day 10. Cartilage explants cultured adjacent to functionalized hydrogels had increased proteoglycan synthesis at day 10 when HB-IGF-1 was adsorbed, but not IGF-1. We conclude that delivery of HBIGF-1 to focal defects in cartilage using self-assembling peptide hydrogels is a promising technique that could aid cartilage repair via enhanced matrix production and integration with native tissue.

\section{Introduction}

I NJURIES TO CARTILAGE are common and have a limited ability to self-repair. ${ }^{1,2}$ One approach to enhancing the native healing response is to deliver proanabolic growth factors to the damaged area using a biomaterial scaffold such that the growth factor is locally delivered at the site of injury and in a sustained manner over time. Insulin-like growth factor 1 (IGF-1) is known to enhance aggrecan production and minimize aggrecan catabolism, making it an exciting candi- date for delivery to cartilage tissue. ${ }^{3-5}$ Unfortunately, unmodified IGF-1 has a short half-life and easily diffuses out of the joint, creating the potential for unwanted side effects. ${ }^{6-8}$

Recently, we have shown that a fusion protein combining the heparin-binding $(\mathrm{HB})$ domain of heparin-binding epidermal growth factor-like growth factor (HB-EGF) and IGF-1, called heparin-binding insulin-like growth factor 1 (HB-IGF1 ), is a promising candidate for sustained, local delivery to cartilage. HB-IGF-1 binds to heparan sulfate (HS) with a $K_{\mathrm{D}}$ of $21 \mathrm{nM}$ and to chondroitin sulfate with a $K_{\mathrm{D}}$ of $160 \mathrm{nM}$, whereas

Parts of this work have been published in the form of abstracts at the Orthopedic Research Society Meeting:

Florine, E.M., Miller, R.E., Lee, R.T., Patwari, P., Grodzinsky, A.J. Heparan sulfate is retained in peptide hydrogel scaffold via ionic interactions: potential for delivery of HB-IGF-1. Abstract presented at the 58th Orthopedic Research Society Meeting, San Francisco, CA, 2012. Abstract no. 1648.

Florine, E.M., Lee, R.T., Patwari, P., Grodzinsky, A.J. HB-IGF-1 Adsorbed to Self-Assembling Peptide Enhances Matrix Production by Encapsulated Chondrocytes and Cocultured Cartilage Explants. Abstract presented at the 59th Orthopedic Research Society Meeting, San Antonio, TX, 2013. Abstract no. 0159.

${ }^{1}$ Biological Engineering, Massachusetts Institute of Technology, Cambridge, Massachusetts.

${ }^{2}$ Rush University Medical Center, Chicago, Illinois.

${ }^{3}$ Brigham Regenerative Medicine Center, Cambridge, Massachusetts.

${ }^{4}$ Brigham and Women's Hospital and Harvard Medical School, Boston, Massachusetts.

This work was performed at the Massachusetts Institute of Technology, Cambridge, MA and the Brigham and Women's Hospital, Cambridge, MA. 
IGF-1 does not bind to either. ${ }^{9}$ The mechanism of binding is thought to be via interactions between the positively charged HB domain of HB-IGF-1 and the negatively charged sulfated glycosaminoglycan (sGAG) chains in cartilage. An intraarticular injection of HB-IGF-1 into the joints of rats showed that the growth factor bound to sGAG-containing articular cartilage and the meniscus, but was not detected in tendon, patella, or muscle. ${ }^{9}$ In addition, sustained bioactivity of a single dose of HB-IGF-1 was demonstrated by stimulation of increased proteoglycan synthesis in cartilage explants 6 days after the washout of unbound growth factor. ${ }^{10}$

Given the promising interactions between HB-IGF-1 and cartilage tissue, we investigated several methods of delivering HB-IGF-1 using the self-assembling peptide (RADA) $)_{4}$, or RAD. We have found self-assembling peptide hydrogels to be advantageous in a rabbit model of cartilage repair, ${ }^{11}$ supportive of chondrogenesis for bone marrow stromal cells, ${ }^{12,13}$ and capable of delivering growth factors in vitro. ${ }^{14}$ The RAD peptide sequence consists of alternating hydrophobic and hydrophilic amino acids, where the hydrophilic amino acids have alternating acidic and basic residues. Since the reversible binding interaction between HB-IGF-1 and chondroitin sulfate chains in cartilage is likely based on charge, we hypothesized that we could take advantage of the charged amino acids in RAD to bind negatively charged HS in complex with HB-IGF-1, or positively charged HB-IGF-1 alone, to deliver HB-IGF-1. We also tested adsorbing HB-IGF-1 to RAD either before self-assembly or after the formation of the hydrogel.

After testing these different methods of adsorbing HBIGF-1 to the peptide, we selected the most promising approach and tested it with chondrocyte-seeded hydrogels and explants cocultured with functionalized hydrogels to see whether we could stimulate proteoglycan synthesis. The ability to deliver a proanabolic growth factor to both the cells encapsulated in the tissue engineering scaffold and the native cartilage tissue surrounding a cartilage defect could be a key step in achieving integrative cartilage repair. Here, we present work showing that adsorption of HB-IGF-1 to RAD self-assembling peptide is a promising method for reaching this goal.

\section{Materials and Methods}

\section{Materials}

$(\text { RADA })_{4}$ self-assembling peptide, hereafter called RAD and also known as PuraMatrix, was a gift from 3DM (Cambridge, MA). Hydrogels were formed at a concentration of $0.5 \%(\mathrm{w} / \mathrm{v})$ for all experiments, consistent with previous literature. $^{12,13,15}$ IGF-1 (Increlex) and HB-IGF-1 proteins were from Ipsen/Biomeasure, Inc. (Milford, MA). Bovine joints were from Research 87 (Marlborough, MA) and HS from bovine kidney (0.88 sulfates/disaccharide $\left.{ }^{16}\right)$ was from Sigma-Aldrich (St. Louis, MO, catalog number H7640). Figure captions describe the number of hydrogels, explants, or animals used in each experiment.

\section{Cartilage explant harvest}

One millimeter thick, $3 \mathrm{~mm}$ diameter middle zone cartilage explants were harvested from the femoropatellar grooves of 1-2 week old bovine calves and allowed to rest for $24 \mathrm{~h}$ in basal medium with low glucose Dulbecco's modified Eagle's medium (Mediatech, Inc., Manassas, VA) supplemented with penicillin, streptomycin, and amphotericin (PSA); 4-(2hydroxyethyl)-1-piperzaineethanesulfonic acid (Invitrogen, Carlsbad, CA); proline (Sigma-Aldrich); ascorbate-2-phosphate (Wake Chemicals, Richmond, VA); and non-essential amino acids (Sigma-Aldrich), as described previously. ${ }^{17}$

\section{Chondrocyte harvest}

Cartilage from the femoral condyles of 1-2 week old bovine calves was collected and digested with pronase for $1 \mathrm{~h}$ and collagenase overnight. ${ }^{18}$ Chondrocytes were filtered from remaining tissue with cell strainers having pore sizes of 70 and $40 \mu \mathrm{m}$ and seeded into hydrogel culture on the same day, as described below in the section "IGF-1 and HB-IGF-1 delivery to peptide-encapsulated bovine chondrocytes."

\section{${ }^{14} \mathrm{C}$-labeling of HB-IGF-1 and IGF-1}

One percent (w/v) stock solutions of IGF-1 and HB-IGF-1 were each dialyzed overnight against $0.3 \mathrm{M}$ sodium phosphate $\mathrm{pH} 7.2$ at $4^{\circ} \mathrm{C}$ in a $2000 \mathrm{MW}$ cutoff cassette. A volume of $13.4 \mu \mathrm{L}$ of $1.25 \mu \mathrm{Ci} / \mu \mathrm{L}\left[1-{ }^{14} \mathrm{C}\right]$-acetic anhydride (American Radiolabeled Chemicals, Inc., St. Louis, MO) dissolved in 1,4-dioxane was added to $125 \mu \mathrm{L}$ of each stock protein and incubated for $30 \mathrm{~min}$ at room temperature. The samples were then redialyzed overnight against sodium phosphate buffer at $4^{\circ} \mathrm{C}$ to remove free radiolabel.

\section{HB-IGF-1 dose-response in cartilage explants}

After a 24-h rest period in basal medium, medium on the cartilage explants was changed and replaced with new basal medium alone, or medium containing $50 \mathrm{nM}$ IGF-1, or 0.5 , 5,50 , or $100 \mathrm{nM} \mathrm{HB}-\mathrm{IGF}-1$ for a culture period of $48 \mathrm{~h}$. Fifty nanomolar IGF-1 was chosen for the IGF-1 concentration because it is known to be effective at stimulating an anabolic response in chondrocytes and cartilage explants. $3,17,19$ At the end of this period, soluble growth factor was removed by washing with phosphate-buffered saline (PBS) and replaced with basal medium. Medium changes with basal medium were performed every 2 days thereafter. For the final $24 \mathrm{~h}$ of culture on day 8 , explants were radiolabeled with $5 \mu \mathrm{Ci} / \mathrm{mL}^{35} \mathrm{~S}$-sulfate (Perkin Elmer, Inc., Waltham, MA) to measure proteoglycan synthesis, as described previously. ${ }^{20}$ Unincorporated radiolabel was removed and explants were weighed wet and digested with Proteinase-K (Roche Applied Science, Indianapolis, IN). Digested samples were analyzed for radiolabel incorporation by liquid scintillation counting and DNA content by Hoechst dye binding. ${ }^{21}$

\section{HS release from acellular RAD peptide hydrogels}

HS was mixed into unassembled RAD peptide solution at a concentration of $5 \mu \mathrm{M}$. One hundred microliter of PBS with PSA was layered on top of $50 \mu \mathrm{L}$ of $\mathrm{HS}$-functionalized RAD to initiate hydrogel self-assembly. ${ }^{22}$ These volumes were chosen to allow predicted levels of HS release from the hydrogel to be detectable. After $15 \mathrm{~min}$, the bath was removed and replaced with a bath of either PBS + PSA ("PBS alone"), PBS + PSA with $1 \mathrm{M} \mathrm{NaCl}$ added ("1 $\mathrm{M} \mathrm{NaCl"),} \mathrm{or}$ 
PBS + PSA with the $\mathrm{pH}$ adjusted to 9 with $\mathrm{NaOH}$ (" $\mathrm{pH} 9$ ") $)$. All hydrogels were cultured at $37^{\circ} \mathrm{C}$. Eighty microliter of the bath from each sample was collected and replaced every $24 \mathrm{~h}$ for days $1-7$. The HS content of the bath was measured by 1.9 -dimethylmethylene blue (DMMB) dye binding. ${ }^{23}$

\section{IGF-1 and HB-IGF-1 retained following adsorption to unassembled peptide}

Initial entrapment efficiency of HB-IGF-1 within the RAD hydrogel was assessed using methods as described previously. ${ }^{24}$ Acellular RAD was mixed with HB-IGF-1 prior to gel assembly to yield a growth factor concentration of $50 \mathrm{ng} / \mu \mathrm{L}(4.92 \mu \mathrm{M})$, or a total of $2.5 \mu \mathrm{g} \mathrm{HB}-\mathrm{IGF}-1$ in $250 \mu \mathrm{g}$ hydrogel. Fifty microliter of functionalized gel was then cast into low-binding microcentrifuge tubes. The gels were allowed to assemble in the tubes for $30 \mathrm{~min}$, after which $500 \mu \mathrm{L}$ of PBS was layered on top of the hydrogel samples to further induce self-assembly. The hydrogels were stored at $37^{\circ} \mathrm{C}$ for $3 \mathrm{~h}$. As a control, $50 \mu \mathrm{L}$ of nonfunctionalized RAD was prepared in the same manner. After $3 \mathrm{~h}$, the PBS was sampled, and the amount of HB-IGF-1 released to PBS during the gel assembly was measured using an IGF-1 enzyme-linked immunosorbent assay (ELISA) known to bind HB-IGF-1 (R\&D Systems, catalog number DY291). The standard curve for the ELISA was prepared using HB-IGF-1 and PBS to match gel/PBS samples. There was no intentional mechanical disruption of the gel. The amount of HB-IGF-1 released from the gel into PBS was subtracted from the amount that was initially pre-mixed into the gel. In this way, the initial entrapment efficiency of HBIGF-1 was calculated.

To test growth factor retention in unassembled peptide, $50 \mathrm{nM}$ IGF-1 or $50 \mathrm{nM}$ HB-IGF-1 was allowed to form a complex with $50 \mathrm{nM}$ HS for $15 \mathrm{~min}$ before mixing with RAD peptides in solution. As additional separate conditions, $50 \mathrm{nM}$ IGF-1 or $50 \mathrm{nM}$ HB-IGF-1 alone were mixed with RAD peptides in solution. Seven hundred fifty microliter of PBS + PSA was layered on top of $50 \mu \mathrm{L}$ of hydrogel samples to initiate self-assembly; hydrogels were then cultured at $37^{\circ} \mathrm{C}$ in low-binding microcentrifuge tubes. These volumes were chosen to match parallel experiments using chondrocyte-seeded hydrogels. The growth factor concentrations correspond to $19 \mathrm{ng}$ IGF-1 or $25 \mathrm{ng}$ HB-IGF-1 being mixed into the peptide. The bath was replaced every $24 \mathrm{~h}$ for 6 days. Hydrogel samples were frozen at each time point after removal of the bath. Determination of growth factor retention was made by western blotting. Hydrogel samples were mechanically disrupted and directly loaded into a $4-12 \%$ Bis-Tris gel (Invitrogen) and run at $200 \mathrm{~V}$ for $45 \mathrm{~min}$. Proteins were transferred to a polyvinylidine fluoride membrane and probed with an anti-IGF-1 antibody (catalog number 9572; Abcam, Cambridge, MA), which recognizes both IGF-1 and HB-IGF-1. ${ }^{10}$

\section{HB-IGF-1 retained following adsorption to preassembled peptide hydrogel}

To determine the initial entrapment efficiency of HBIGF-1 to preassembled peptide, $50 \mu \mathrm{L}$ of RAD hydrogel was assembled overnight at $37^{\circ} \mathrm{C}$ in a bath of PBS + PSA. ${ }^{14} \mathrm{C}$ HB-IGF-1 was added to the bath for a final concentration of $3.28 \mu \mathrm{M}$ of radiolabeled protein in a bath volume of $300 \mu \mathrm{L}$.
Following overnight incubation at $37^{\circ} \mathrm{C}$, the uptake baths were removed, and the hydrogels were gently rinsed twice with PBS + PSA. A fresh bath of $500 \mu \mathrm{L}$ of PBS + PSA was added to the hydrogel, and protein was allowed to desorb from the hydrogel for $3 \mathrm{~h}$. Following desorption, the bath was removed from the hydrogel, and the hydrogel was mechanically disrupted. Radiolabeled protein in the desorption baths and mechanically disrupted hydrogels were measured by liquid scintillation counting. Entrapment efficiency was determined as the amount of protein remaining in the hydrogel following the 3-h desorption, expressed as nanograms of protein retained/micrograms of hydrogel.

To test growth factor retention in preassembled hydrogels, $50 \mu \mathrm{L}$ RAD hydrogels with or without $50 \mathrm{nM}$ HS mixed in were allowed to assemble for $24 \mathrm{~h}$ in $750 \mu \mathrm{L}$ PBS + PSA at $37^{\circ} \mathrm{C}$ in low-binding microcentrifuge tubes. After $24 \mathrm{~h}$, the bath was replaced with PBS + PSA with $50 \mathrm{nM} \mathrm{HB-IGF-1,}$ which provides a total of $380 \mathrm{ng}$ of HB-IGF-1 in the medium to potentially bind to the peptide. Twenty-four hours after addition of the HB-IGF-1 bath, soluble growth factor was washed out of the cultures using PBS and the bath was replaced with PBS + PSA. Hydrogel samples were frozen after bath removal every $24 \mathrm{~h}$ through day 8 . Growth factor retention was assessed by western blotting, as described above.

\section{Release of ${ }^{14} \mathrm{C}-\mathrm{HB}-\mathrm{IGF}-1$ and ${ }^{14} \mathrm{C}$-IGF-1 following adsorption to unassembled peptide}

To determine a quantitative release profile, ${ }^{14} \mathrm{C}-\mathrm{IGF}-1$ or ${ }^{14} \mathrm{C}$-HB-IGF-1 was mixed into unassembled RAD peptide solution at a concentration of $615 \mathrm{nM}$. Thirty microliters of PBS with PSA was layered on top of $50 \mu \mathrm{L}$ of HB-IGF-1or IGF-1-functionalized RAD to initiate hydrogel selfassembly. An additional $470 \mu \mathrm{L}$ of PBS + PSA was added to the bath to reach a final volume of $500 \mu \mathrm{L}$, and the hydrogels were incubated at $37^{\circ} \mathrm{C}$. Two hundred fifty microliters of the bath from each sample were collected and replaced every $24 \mathrm{~h}$ during days $1-8$, and every $48 \mathrm{~h}$ between days 8 and 12 . At day 12 , the baths were completely replaced with $10 \times$ PBS. On day 14 , the $10 \times$ PBS baths were removed and saved, and the hydrogels were mechanically disrupted and saved. Radiolabeled protein in the bath and mechanically disrupted hydrogels were measured by liquid scintillation counting.

\section{IGF-1 and HB-IGF-1 delivery to peptide-encapsulated bovine chondrocytes}

Bovine chondrocytes were encapsulated in RAD at a density of 30 million cells/mL. The RAD was either unaltered (for "Basal," "IGF soluble," and "HB soluble", conditions), or had one of the following adsorbed to RAD prior to cell encapsulation: $50 \mathrm{nM}$ IGF-1 (for "IGF premix" condition) or $50 \mathrm{nM}$ HB-IGF-1 (for "HB pre-mix" condition). See Table 1 for a summary of the culture conditions. On day 0 , the cell/hydrogel mixture was cast as $6 \mathrm{~mm}$ diameter, $1.5 \mathrm{~mm}$ thick disks into agarose rings preequilibrated in the basal medium described above, as described previously. ${ }^{12}$ Some hydrogels were cast into $50 \mathrm{mM}$ tris(hydroxymethyl)aminomethane (Tris) and $1 \mathrm{mM} \mathrm{CaCl} 2$ at the time of casting to measure day-0 DNA levels. The hydrogel disks were cultured in basal medium for $24 \mathrm{~h}$ to 
Table 1. Functionalized RAD Hydrogel and Medium Conditions for Experiments with RAD Peptide Seeded with Bovine Chondrocytes and Cartilage Explants

\begin{tabular}{llcc}
\hline Condition & \multicolumn{1}{c}{ HB/IGF source } & $H S$ & $\begin{array}{c}\text { Time soluble GF } \\
\text { present (days) }\end{array}$ \\
\hline Basal & None & None & N/A \\
IGF soluble & Soluble in media & None & $1-10$ \\
HB soluble & Soluble in media & None & $1-10$ \\
IGF-pre-mix & Adsorbed to peptides prior to gel assembly & None & 0 \\
HB pre-mix & Adsorbed to peptides prior to gel assembly & None & 0 \\
\hline
\end{tabular}

Refer to Figure 6 for time course of experimental procedure.

HB, heparin-binding; IGF, insulin-like growth factor; HS, heparan sulfate; GF, growth factor; N/A, not applicable.

allow peptide assembly. After $24 \mathrm{~h}$, on day 1 , a medium change was performed on all hydrogels. Hydrogels for the "IGF soluble" and "HB soluble" conditions received $50 \mathrm{nM}$ IGF-1 and HB-IGF-1, respectively, in the medium through the entire culture period. Hydrogels for the "Basal," "IGF pre-mix," and "HB pre-mix" conditions received basal medium for the entire culture duration. Hydrogel samples were cultured up to 10 days with medium changes every 2 days.

For the final $24 \mathrm{~h}$ of culture, hydrogels were radiolabeled with $5 \mu \mathrm{Ci} / \mathrm{mL}^{35} \mathrm{~S}$-sulfate and $10 \mu \mathrm{Ci} / \mathrm{mL}^{3} \mathrm{H}$-proline (Perkin Elmer, Inc.) to measure proteoglycan and total protein synthesis, respectively. Unincorporated radiolabel was removed and hydrogels were weighed wet, lyophilized, weighed dry, and digested with Proteinase-K (Roche Applied Science), as described previously. ${ }^{12}$ Digested samples were analyzed for radiolabel incorporation by liquid scintillation counting, for $s G A G$ content by DMMB dye binding, ${ }^{23}$ for DNA content by Hoechst dye binding, ${ }^{21}$ and hydroxyproline content as a measure of total collagen by reaction with $p$-dimethylaminobenzaldehyde. $^{25}$

\section{IGF-1 and HB-IGF-1 delivery to explants cultured with acellular functionalized peptide}

Bovine cartilage explants were harvested and placed in basal medium to rest. One day after the harvest, $100 \mu \mathrm{L}$ RAD hydrogels were cast into $2 \mathrm{~mL}$ cryo-tubes and assembled with $300 \mu \mathrm{L}$ of basal medium. The hydrogel and medium conditions, and the schedule of medium changes, were identical to those described for the bovine chondrocyte experiment described above. The cartilage explants received new basal medium on day 1 and were added to the cryotubes with the acellular peptide on day 2 .

Explants were radiolabeled with $5 \mu \mathrm{Ci} / \mathrm{mL}{ }^{35} \mathrm{~S}$-sulfate (Perkin Elmer, Inc.) for the final $24 \mathrm{~h}$ of culture to measure proteoglycan synthesis. Explants were washed to remove unincorporated radiolabel, weighed wet, and digested with Proteinase-K (Roche Applied Science). Digested samples were analyzed for radiolabel incorporation by liquid scintillation counting and DNA content was measured by Hoechst dye binding.

\section{Statistics}

A general linear model with medium condition as an independent variable was used to analyze HB-IGF-1 doseresponse data. HS release data were rank transformed and analyzed using a general linear model with medium condi- tion and day as independent variables. For chondrocyteseeded hydrogel experiments, a one-way analysis of variance with culture condition as an independent variable was used to analyze each time point from each of two replicate experiments separately for sGAG per DNA, proteoglycan synthesis, protein synthesis, and hydroxyproline content data. Statistical symbols are those that were consistent between the two experiments. A Kolmogorov-Smirnov test was used to test for normality and data were transformed as necessary for all the statistical tests above. A linear mixed model with animal as a random factor and culture condition as an independent variable was used to analyze data from the three functionalized hydrogel-cartilage explant coculture experiments together. Residuals were analyzed and data transformed as needed to ensure normality. Tukey post hoc tests with $p<0.05$ were used to evaluate statistical significance for all pairwise comparisons. Systat 12 software (Systat, Chicago, IL) was used for all statistical analyses.

\section{Results}

\section{HB-IGF-1 dose-response in cartilage explants}

To determine a dose of HB-IGF-1 for use in later experiments, a dose-response study was first performed on cartilage explants. Explants received medium with either soluble IGF-1 or HB-IGF-1 at various doses for $48 \mathrm{~h}$ and then unbound growth factor was removed by washing with PBS. Six days later, explants were radiolabeled to measure proteoglycan synthesis (Fig. 1A). Six days after removing soluble growth factor, the $50 \mathrm{nM}$ IGF-1 condition ("IGF 50 ") was not statistically different from the no IGF (" 0 ") control (Fig. 1B), consistent with previous reports. ${ }^{10}$ Doses of 5, 50, and $100 \mathrm{nM}$ HB-IGF-1 showed significantly greater proteoglycan synthesis than the no IGF control and the dose of $0.5 \mathrm{nM}$ HB-IGF-1. There was a trend on increasing proteoglycan synthesis as the HB-IGF-1 dose increased, although 5, 50, and $100 \mathrm{nM} \mathrm{HB}-\mathrm{IGF}-1$ were not statistically distinct. As the peak effect of HB-IGF-1 was seen with $50 \mathrm{nM}$ HB-IGF-1, this dose was chosen for future experiments.

\section{HS release from acellular RAD peptide hydrogel}

Because of the strong interaction between HS and HBIGF-1, we hypothesized that including HS in the peptide gel could provide additional binding sites for the growth factor and enhance the longevity of its delivery. As an initial step toward this goal, we investigated whether HS alone could be retained in the peptide. HS was adsorbed to unassembled 


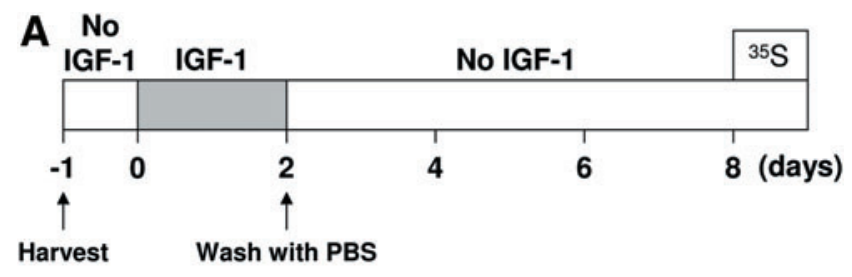

B Normalized Proteoglycan Synthesis

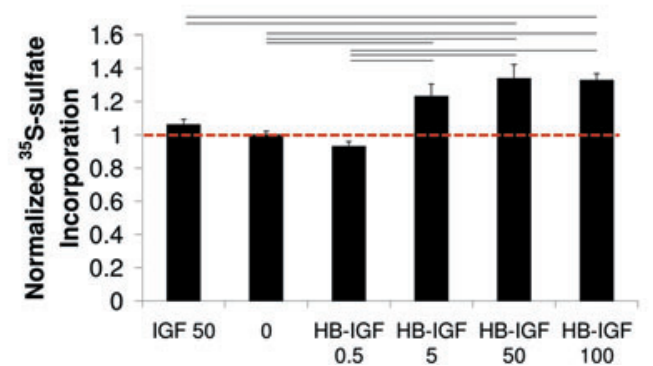

FIG. 1. Heparin-binding insulin-like growth factor 1 (HBIGF-1) dose-response in cartilage explants at day 8 following wash-out procedure. (A) Timeline of experimental procedure. Explants were treated with $50 \mathrm{nM}$ IGF-1, or 0.5 , 5 , 50, or $100 \mathrm{nM}$ HB-IGF-1 from day 0 to 2. (B) Proteoglycan synthesis normalized to DNA content $(\mu \mathrm{g})$ and the no-growth factor ("0") condition (dashed line) at day 8. Values are mean \pm standard error of the mean (SEM). $N=6$ cartilage disks per condition, one animal. Solid lines indicate significant difference between two conditions, $p<0.05$. Color images available online at www.liebertpub.com/tea

RAD peptides in solution and cultured in a bath of either PBS alone, PBS + $1 \mathrm{M} \mathrm{NaCl} \mathrm{("1} \mathrm{M} \mathrm{NaCl"),} \mathrm{or} \mathrm{PBS} \mathrm{with} \mathrm{the}$ $\mathrm{pH}$ adjusted to 9 by $\mathrm{NaOH}$ (" $\mathrm{pH} 9$ 9"). Release of HS from the peptide was measured over a 7-day period (Fig. 2). In PBS alone, $<15 \%$ of the total HS was released from the RAD by day 7 . The addition of $1 \mathrm{M} \mathrm{NaCl}$ or alteration of the $\mathrm{pH}$ to 9 resulted in increased release of HS compared with PBS alone, with the $1 \mathrm{M} \mathrm{NaCl}$ bath causing more release than the $\mathrm{pH} 9$ bath.

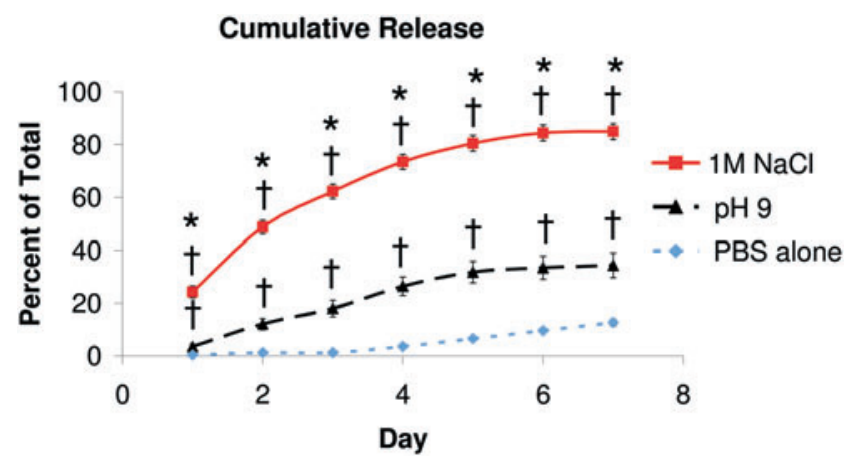

FIG. 2. Heparan sulfate (HS) release from acellular peptide hydrogels. Peptide with $5 \mu \mathrm{M}$ HS cultured in $100 \mu \mathrm{L}$ bath of phosphate-buffered saline (PBS), PBS with 1M $\mathrm{NaCl}$ (" $1 \mathrm{M} \mathrm{NaCl}$ ), or PBS with a $\mathrm{pH}$ of 9 (adjusted using $\mathrm{NaOH}$, " $\mathrm{pH} 9$ 9"). Values are mean \pm SEM. $N=6-8$, except at day 6 where $n=2$. ${ }^{\dagger}$ Versus PBS alone, *versus $\mathrm{pH} 9$, $p<0.05$. Color images available online at www.liebertpub .com/tea
IGF-1 and HB-IGF-1 retained following adsorption

to unassembled peptide

To test the initial entrapment efficiency of HB-IGF-1 in unassembled peptide, $2.5 \mu \mathrm{g}$ HB-IGF-1 was mixed with $250 \mu \mathrm{g}$ unassembled RAD and HB-IGF-1 release to the PBS bath was measured after $3 \mathrm{~h}$, similar to methods described previously. ${ }^{24}$ We found that the RAD peptide retains $\sim 9 \mathrm{ng}$ HB-IGF-1 per microgram of peptide. We next tested whether HS improved retention of HB-IGF-1 in the peptide. To test this, $50 \mathrm{nM}$ IGF-1 or HB-IGF-1 with or without $50 \mathrm{nM}$ HS was mixed into unassembled RAD, PBS was added to initiate assembly, and the PBS bath was then changed daily. Each day, hydrogels were frozen after the bath was removed and their growth factor content assessed by western blotting. HB-IGF-1 was strongly retained in the hydrogel with or without HS for the entire culture duration (Fig. 3A). IGF-1 was also retained for the entire culture duration with or without HS (Fig. 3B).

\section{HB-IGF-1 retained following adsorption to preassembled peptide hydrogel}

To test the initial entrapment efficiency of HB-IGF-1 to preassembled hydrogels, ${ }^{14} \mathrm{C}$-labeled HB-IGF-1 was added to a $300 \mu \mathrm{L}$ PBS bath at a concentration of $3.28 \mu \mathrm{M}$. After $24 \mathrm{~h}$ at $37^{\circ} \mathrm{C}$, the bath was removed, hydrogels were rinsed with PBS, and a $500 \mu \mathrm{L}$ bath of fresh PBS was added. After $3 \mathrm{~h}$, the amount of radiolabeled protein in the bath and the mechanically disrupted gel were measured. About $0.57 \mathrm{ng}$ of HB-IGF-1 per microgram of peptide was retained. To investigate retention over a longer time, $50 \mathrm{nM}$ HB-IGF- 1 was allowed to adsorb for $24 \mathrm{~h}$ to preassembled hydrogels of RAD alone or RAD with $50 \mathrm{nM}$ HS mixed in prior to assembly. Soluble growth factor was then washed out of the culture using PBS (Fig. 4A). Time points were taken daily to assess retention of HB-IGF-1 by western blotting. HB-IGF-1 was retained in RAD (Fig. 4B) and RAD functionalized with HS throughout the culture period (Fig. 4C). Lower levels of

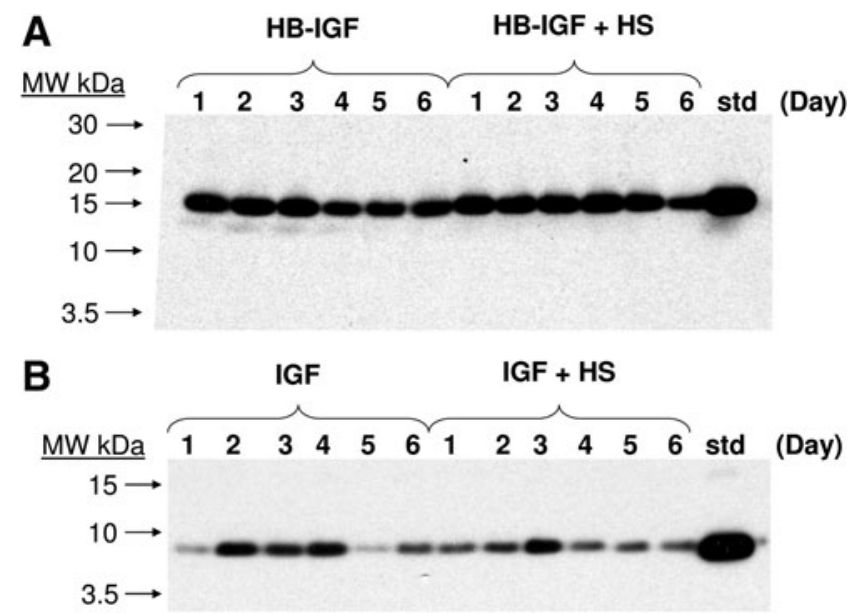

FIG. 3. Growth factor retention when mixed into initially unassembled acellular peptide. Western blots of (A) HBIGF-1 and (B) IGF-1 retained in peptide with or without HS on days 1 through 6 , using an anti-IGF-1 antibody. Std is a standard of $5 \mathrm{ng}$ HB-IGF-1 or IGF-1 for (A) and (B), respectively. 


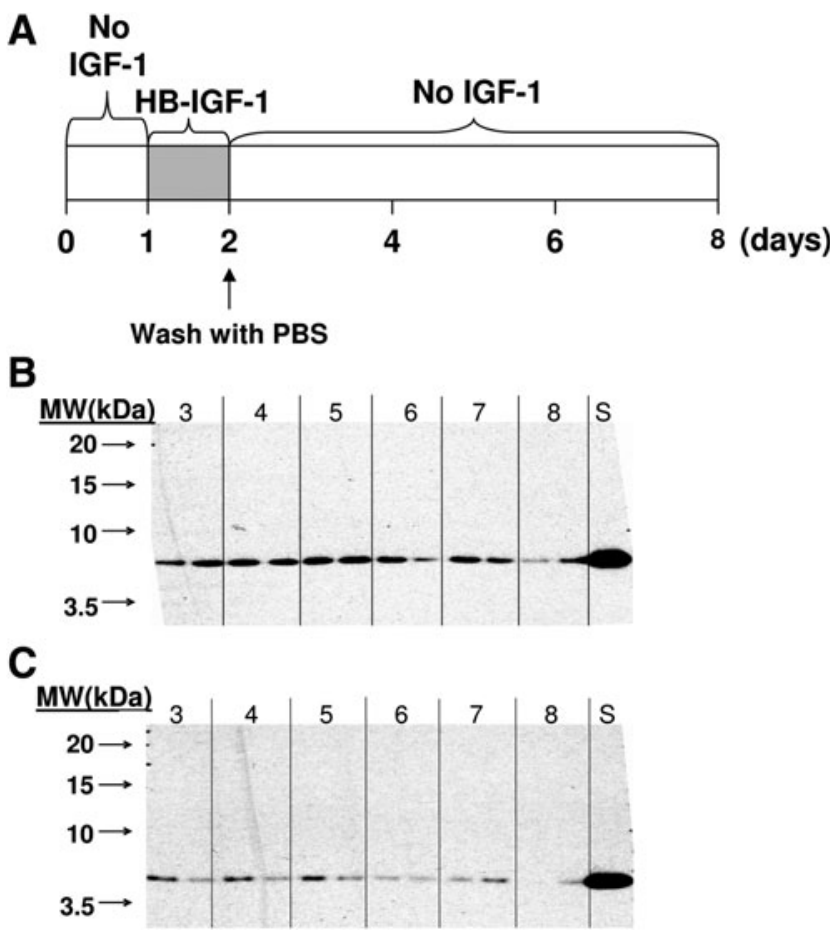

FIG. 4. HB-IGF-1 retention when adsorbed to preassembled acellular peptide. (A) Time course of experimental procedure where peptide $\pm \mathrm{HS}$ assembled from day 0 to 1 and HB-IGF-1 was absorbed to peptide from day 1 to 2 . Western blots of (B) HB-IGF-1 retained by days 3-8 after adsorption to peptide alone, and (C) HB-IGF-1 retained by days 3-8 after adsorption to peptide mixed with $\mathrm{HS}$ prior to assembly. $\mathrm{S}$ is a standard of $5 \mathrm{ng}$ HB-IGF-1. Two lanes for each time point are repeats of the same condition.

protein were seen at day 8 in both conditions, perhaps suggesting release of the adsorbed HB-IGF-1.

Based on the results in Figures 3 and 4 we concluded that HS was not needed to retain HB-IGF-1 within the peptide hydrogel, so these conditions were not tested in the cellbased experiments. Furthermore, since assembling the peptide prior to adsorbing the HB-IGF-1 resulted in a lower initial entrapment efficiency and the only practical method of delivering the growth factor in vivo using the peptide is to mix them together prior to peptide assembly, we proceeded with the method of mixing the growth factor into the unassembled peptide solution.

\section{Release of ${ }^{14} \mathrm{C}-\mathrm{HB}-\mathrm{IGF}-1$ and ${ }^{14} \mathrm{C}$-IGF-1 following adsorption to unassembled peptide}

To assess the release profile of HB-IGF-1 as compared to IGF-1, we radiolabeled both growth factors with ${ }^{14} \mathrm{C}$ and loaded them at a concentration of $615 \mathrm{nM}$ into unassembled peptide. The release to a PBS bath was measured over the following 12 days (Fig. 5). Both growth factors were released from the peptide hydrogel, with the ${ }^{14} \mathrm{C}-\mathrm{IGF}-1$ releasing from the peptide at a faster rate than the ${ }^{14} \mathrm{C}-\mathrm{HB}$ IGF-1 over the first 4 days. Both growth factors had a slower rate of release in the second half of the time course, with the IGF curve approaching $100 \%$ release by day 7 and the HB-IGF curve at $65 \%$ of the material released by day 12 .

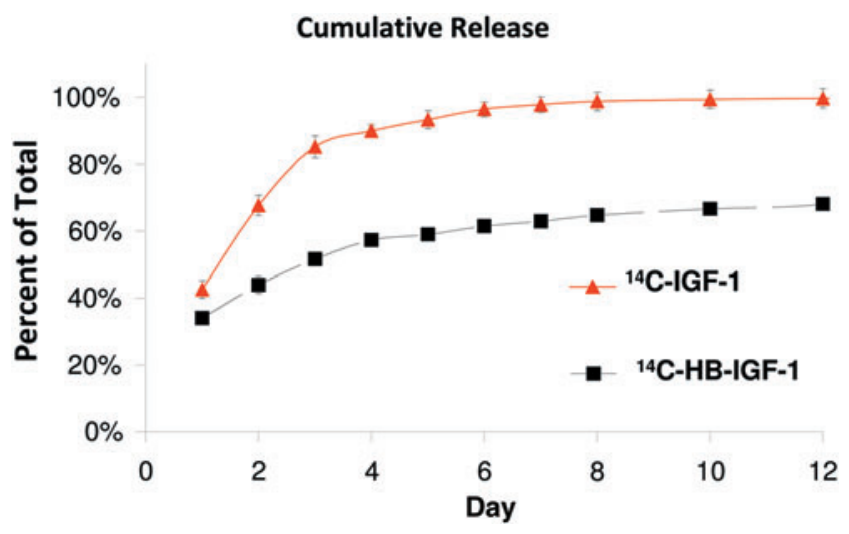

FIG. 5. ${ }^{14} \mathrm{C}$-IGF- 1 and ${ }^{14} \mathrm{C}-\mathrm{HB}-\mathrm{IGF}-1$ release from acellular peptide scaffold. Peptide with $615 \mathrm{nM}$ labeled growth factor was cultured in a $500 \mu \mathrm{L}$ bath of PBS. Values are mean \pm standard deviation. $N=4$. Color images available online at www.liebertpub.com/tea

These results are consistent with the western blots shown in Figure 3.

\section{IGF-1 and HB-IGF-1 delivery to peptide-encapsulated bovine chondrocytes}

We next tested the ability of HB-IGF-1 adsorbed to RAD to stimulate bovine chondrocytes encapsulated within the peptide hydrogel. For the "HB pre-mix" and "IGF premix" conditions, $50 \mathrm{nM}$ HB-IGF-1 or IGF-1, respectively, were adsorbed to unaltered RAD prior to peptide selfassembly and cultured in basal medium (Fig. 6). Unaltered RAD was cultured in basal medium as a negative control ("Basal") or in medium containing $50 \mathrm{nM}$ soluble IGF-1 or HB-IGF-1 throughout the 10-day culture period as positive controls ("IGF soluble" and "HB soluble," respectively). Table 1 summarizes these peptide and medium conditions.

sGAG content normalized to DNA content was not significantly different between any conditions at day 6 (Fig. 7A). sGAG and DNA content are individually shown in Supplementary Figure 1 (Supplementary Data are available online at www.liebertpub.com/tea). The soluble conditions, HB pre-mix, and IGF pre-mix were significantly greater than the Basal condition at day 10. HB pre-mix showed lower proteoglycan synthesis at day 6 than the HB soluble condition (Fig. 7B). By day 10, the soluble conditions were significantly higher than the Basal condition. Protein synthesis for HB pre-mix and IGF pre-mix were lower than IGF soluble and HB soluble at day 6 (Fig. 7C). At day 10, the soluble conditions exceeded the Basal condition, identical to proteoglycan synthesis. All conditions showed increased hydroxyproline content compared with the Basal condition at day 6 and 10 (Fig. 7D).

\section{IGF-1 and HB-IGF-1 delivery to explants cultured with acellular functionalized peptide}

To ensure that the growth factor adsorbed to the RAD was bioactive and bioavailable, and also to test the hypothesis that HB-IGF-1 could be released from the peptide to stimulate nearby cartilage, we repeated the above experiment with acellular functionalized peptides cocultured with 


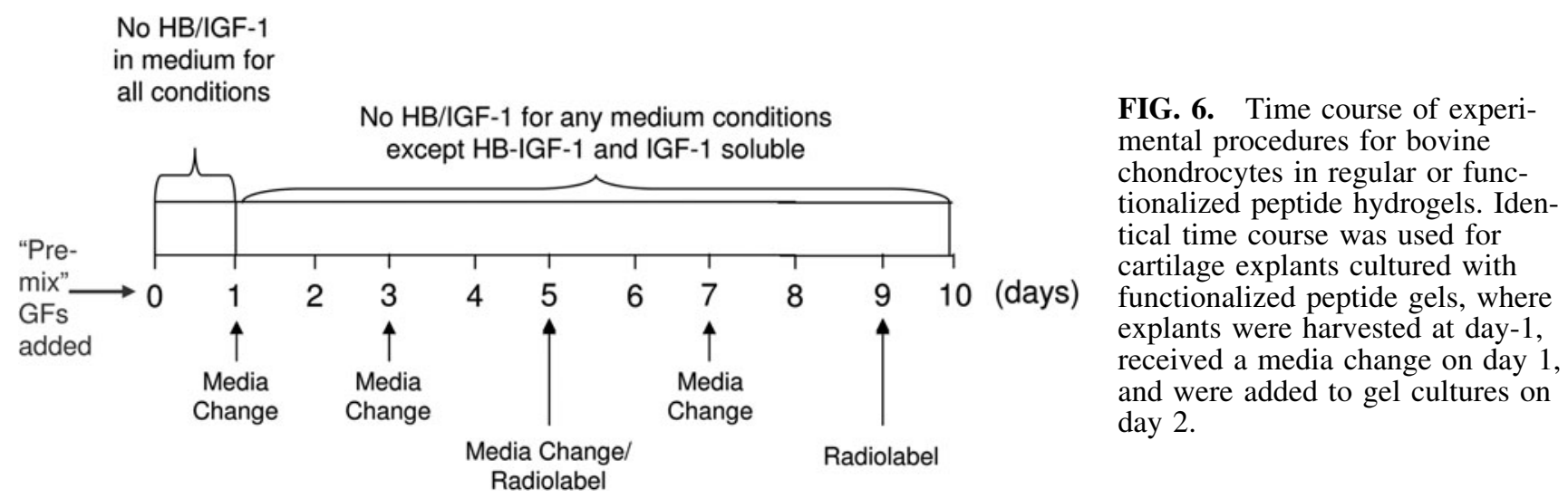

bovine cartilage explants. Explants from all conditions showed higher proteoglycan synthesis compared with the Basal condition at day 6 (Fig. 8). By day 10, the HB-IGF-1 condition maintained increased proteoglycan synthesis levels compared with the Basal condition. In contrast, the IGF pre-mix condition showed significantly lower synthesis levels compared with IGF soluble and was not statistically distinct from Basal at day 10 .

\section{Discussion}

We tested the hypotheses that HB-IGF-1 adsorbed to RAD could stimulate increased sGAG accumulation in chondrocyte-seeded hydrogels and increased proteoglycan synthesis in cartilage explants cocultured with growth factor functionalized hydrogels. We established a dose-response of HB-IGF-1 and confirmed that IGF-1 does not stimulate increased proteoglycan synthesis in cartilage explants after washing unbound growth factor out of the culture. ${ }^{10} \mathrm{We}$ found that HS could be retained in RAD when added to unassembled RAD. Since the HS was minimally released into a bath of PBS at $\mathrm{pH} 7.4$, but could be released by increasing the $\mathrm{pH}$ or adding salt to the bath (Fig. 2), we conclude that HS is retained in RAD via ionic interactions, for example, the positively charged amino acids. Given the strong retention of HS in RAD and the nanomolar binding constant between HS and HB-IGF-1, we hypothesized that HS could be used to retain HB-IGF-1 in the peptide. Because the interaction between HS and RAD was based on charge, we also thought that positively charged HB-IGF-1 might be retained by the negatively charged amino acids in RAD by charge interactions without the presence of HS. We therefore pursued both of these delivery methods. HB-IGF-1 was retained in RAD with or without HS and regardless of

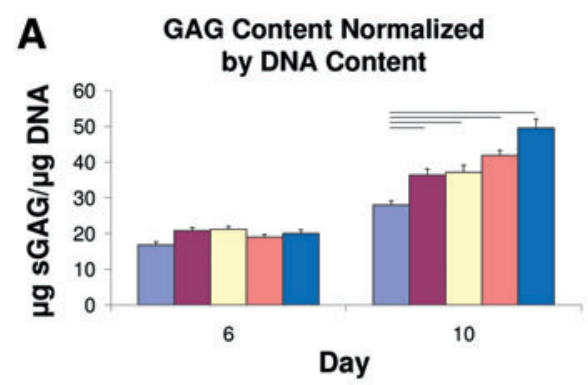

C

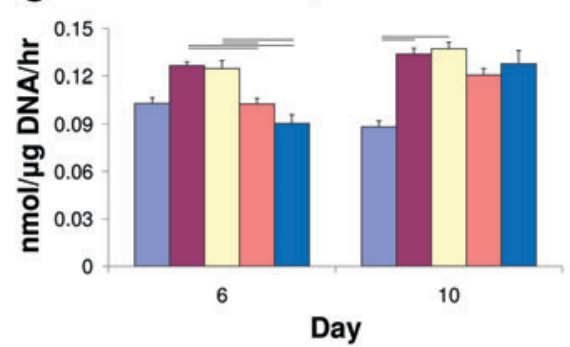

E

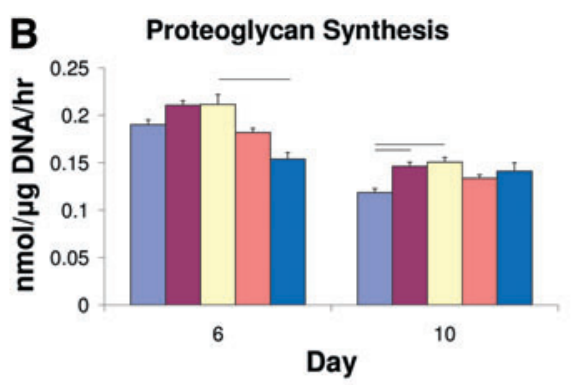

D

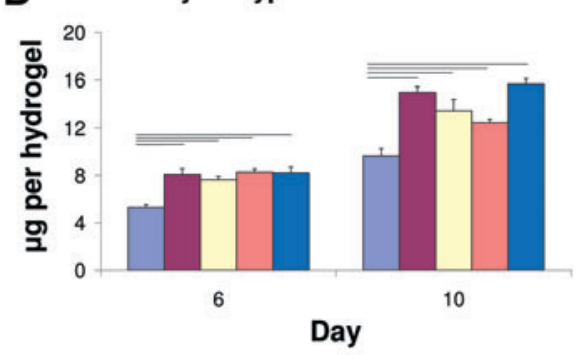

FIG. 7. Extracellular matrix content and biosynthesis by bovine chondrocytes encapsulated within peptide hydrogels. (A) Sulfated glycosaminoglycan (sGAG) content normalized by DNA content, (B) proteoglycan synthesis, (C) protein synthesis, (D) hydroxyproline content, and (E) key of culture conditions. Values are mean \pm SEM. $N=5-6 \times 2$ experiments with cells from different animal donors; data from one experiment shown above. Line indicates significant difference between two conditions consistent between both experiments, $p<0.05$. See Figure 6 for time course of experimental procedure. Color images available online at www.liebertpub.com/tea 

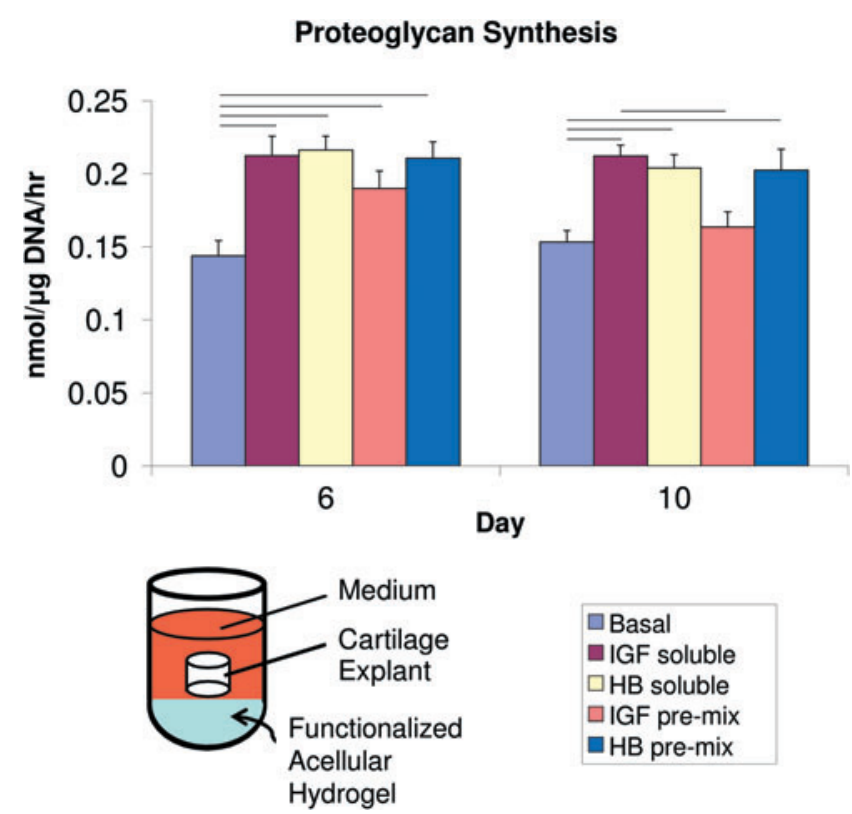

FIG. 8. Proteoglycan synthesis within cartilage explants cultured with growth factor-functionalized acellular peptide hydrogel. Data normalized to DNA content. Values are mean \pm SEM. $N=11-12$ (3-4 explants $\times 3$ animals). Line indicates significant difference between two conditions, $p<0.05$. Color images available online at www.liebertpub.com/tea

whether it was adsorbed to the peptide before or after it was assembled. HB-IGF-1 was found to release more slowly than untargeted IGF-1 when mixed into unassembled peptide (Fig. 5).

Having established that HB-IGF-1 could be retained in the peptide, we tested whether the growth factor could stimulate cells encapsulated within the hydrogel and chondrocytes within adjacent cartilage explants. We found that adsorbing HB-IGF-1 or IGF-1 to the peptide in solution, before it was assembled, increased sGAG content normalized by DNA content in chondrocyte-seeded hydrogels compared with the Basal condition at day 10. Hydroxyproline content was also enhanced compared with the Basal condition at day 6 and 10. Both proteoglycan and protein synthesis levels for the HB pre-mix condition were lower than the soluble conditions at day 6 , but this did not correlate with the sGAG/DNA and hydroxyproline results. This could be explained by a release of growth factor from the functionalized peptide between the day 6 and 10 time points.

In the explant experiment (Fig. 8), there was increased proteoglycan synthesis for explants cocultured with all growth factor functionalized peptide conditions compared with the Basal peptide at day 6 . While IGF-1 did not provide a sustained increase in proteoglycan synthesis in cartilage explants compared to the basal control at day 10, the presence of a binding arm (HB-IGF-1) resulted in sustained stimulation, analogous to results found previously where HB-IGF-1 was delivered in the medium to cartilage explants. ${ }^{10}$ As the N-terminal tri-peptide sequence of IGF-1 is the region associated with the IGF-1 binding to IGF-binding proteins (IGFBPs) in native tissues, ${ }^{26}$ the addition of the HB domain at this $\mathrm{N}$-terminal region of IGF-1 may make the HB-IGF-1 fusion protein unable to bind to IGFBPs. As a result, it is possible that the presence of IGFBPs in cartilage explants $^{27}$ would sequester more IGF-1 than HB-IGF-1. Interestingly, we observed that explants could be stimulated by certain growth factor-functionalized conditions (Fig. 8) that were not as clearly stimulatory for encapsulated chondrocytes in vitro (Fig. 7). We believe this may be due to the fact that there is less extracellular GAG-rich aggrecan surrounding the newly seeded chondrocytes to capture HBIGF-1 released by the hydrogel.

While radiolabel assays have better sensitivity than other methods of detection, at day 12 only $65 \%$ of the radiolabeled HB-IGF-1 was released to the PBS bath. We attempted to recover growth factor remaining in the peptide by adding $10 \times$ PBS and then mechanically disrupting the gel itself to measure the remaining HB-IGF-1 directly, but these procedures only detected an additional $10 \%$ of the material. We hypothesize that the HB-IGF-1 is generally more "sticky" due to the charged HB domain, resulting in small losses throughout the course of the experiment that account for the remaining material.

Ongoing studies focus on the use of radiolabeled HBIGF-1 to characterize transport within hydrogels and native cartilage, including diffusion and binding in space and time. This approach may also be useful in vivo to demonstrate release of HB-IGF-1 from the hydrogel and binding to surrounding cartilage tissue. Additionally, in an experiment using a similar peptide, $\mathrm{KLD}^{12}$, where the hydrogel was cast into an annulus of cartilage tissue, $62 \%$ of the HB-IGF-1 loaded into the gel was recovered from the cartilage annulus after 9 days in culture, whereas only $4 \%$ of the initially loaded growth factor was recovered from the culture medium. Based on the medium volume and explant-medium geometry, these results suggest that the majority of the growth factor diffused laterally into the cartilage rather than being released to the medium and then adsorbed to the cartilage. Future work also remains for understanding how HS and HB-IGF-1 interact with unassembled and assembled RAD fibers. Though we hypothesize that the interactions are based on charge, the stoichiometry and how the interactions might change as self-assembly proceeds are as yet unknown.

We note that the work presented here utilized immature bovine cartilage for explants and chondrocytes due to availability of tissue. Confirmation of the translational value of these results using adult bovine tissue and importantly, mature human cartilage, would be very valuable, as important differences have been shown between cells from different species and ages of donors. ${ }^{13,28-31}$ In the clinical application, the peptide would likely be used to fill in a cartilage defect following microfracture, so the cell population would be more similar to bone marrow stromal cells than chondrocytes.

We have shown that HB-IGF-1 adsorption to RAD selfassembling peptide hydrogels is a promising method for delivering the proanabolic, anticatabolic growth factor IGF-1 to cells encapsulated within hydrogels and to adjacent cartilage tissue. In terms of potential clinical usage, mixing HBIGF with peptide solution prior to hydrogel self-assembly is a simple one step procedure for delivering a growth factor using a hydrogel. The finding that a single dose of HB-IGF-1 mixed into the unassembled peptide at day 0 was still stimulating statistically significant enhancement of proteoglycan synthesis in adjacent cartilage explants compared with Basal 
control at day 10 demonstrates the utility of this approach (Fig. 8). We therefore suggest that HB-IGF-1 delivered via the peptide hydrogel to adjacent cartilage at the site of a focal defect in vivo would be capable of binding to sGAG chains within the cartilage and thereby enabling sustained bioactivity. The hypothesis that HB-IGF-1 would enhance the integration of peptide hydrogel-mediated neocartilage to adjacent native cartilage at a defect interface will be tested in future animal studies. Taken together, we believe that functionalized self-assembling peptide hydrogels provide a promising tissue engineering scaffold for enhancing cartilage repair.

\section{Acknowledgments}

We wish to acknowledge the following funding sources: National Science Foundation Graduate Research Fellowship, Whitaker Health Sciences Fund Fellowship, National Institutes of Health Grants EB003805 and AR060331, Massachusetts Life Science Center, Biomeasure, Inc.

\section{Disclosure Statement}

Brigham and Women's Hospital has filed for patents pertaining to the described HB-IGF-1 technology, listing P.P. and R.T.L. as inventors. Dr. Grodzinsky has equity in 3D Matrix, Ltd., Japan. The other authors of this work have no competing financial interests.

\section{References}

1. Ahmed, T.A., and Hincke, M.T. Strategies for articular cartilage lesion repair and functional restoration. Tissue Eng Part B Rev 16, 305, 2010.

2. Anderson, D.D., Chubinskaya, S., Guilak, F., Martin, J.A., Oegema, T.R., Olson, S.A., and Buckwalter, J.A. Post-traumatic osteoarthritis: improved understanding and opportunities for early intervention. J Orthop Res 29, 802, 2011.

3. Tyler, J.A. Insulin-like growth factor 1 can decrease degradation and promote synthesis of proteoglycan in cartilage exposed to cytokines. Biochem J 260, 543, 1989.

4. Luyten, F.P., Hascall, V.C., Nissley, S.P., Morales, T.I., and Reddi, A.H. Insulin-like growth factors maintain steady-state metabolism of proteoglycans in bovine articular cartilage explants. Arch Biochem Biophys 267, 416, 1988.

5. McQuillan, D.J., Handley, C.J., Campbell, M.A., Bolis, S., Milway, V.E., and Herington, A.C. Stimulation of proteoglycan biosynthesis by serum and insulin-like growth factor-I in cultured bovine articular cartilage. Biochem $\mathbf{J}$ 240, 423, 1986.

6. Laron, Z. Somatomedin-1 (recombinant insulin-like growth factor-1): clinical pharmacology and potential treatment of endocrine and metabolic disorders. BioDrugs 11, 55, 1999.

7. Chan, J.M., Stampfer, M.J., Giovannucci, E., Gann, P.H., Ma, J., Wilkinson, P., Hennekens, C.H., and Pollak, M. Plasma insulin-like growth factor-I and prostate cancer risk: a prospective study. Science 279, 563, 1998.

8. Jabri, N., Schalch, D.S., Schwartz, S.L., Fischer, J.S., Kipnes, M.S., Radnik, B.J., Turman, N.J., Marcsisin, V.S., and Guler, H.P. Adverse effects of recombinant human insulin-like growth factor I in obese insulin-resistant type II diabetic patients. Diabetes 43, 369, 1994.

9. Miller, R.E., Grodzinsky, A.J., Cummings, K., Plaas, A.H., Cole, A.A., Lee, R.T., and Patwari, P. Intraarticular injection of heparin-binding insulin-like growth factor 1 sustains delivery of insulin-like growth factor 1 to cartilage through binding to chondroitin sulfate. Arthritis Rheum 62, 3686, 2010.

10. Tokunou, T., Miller, R., Patwari, P., Davis, M.E., Segers, V.F., Grodzinsky, A.J., and Lee, R.T. Engineering insulinlike growth factor-1 for local delivery. FASEB J 22, 1886, 2008.

11. Miller, R.E., Grodzinsky, A.J., Vanderploeg, E.J., Lee, C., Ferris, D.J., Barrett, M.F., Kisiday, J.D., and Frisbie, D.D. Effect of self-assembling peptide, chondrogenic factors, and bone marrow-derived stromal cells on osteochondral repair. Osteoarthritis Cartilage 18, 1608, 2010.

12. Kopesky, P.W., Vanderploeg, E.J., Sandy, J.S., Kurz, B., and Grodzinsky, A.J. Self-assembling peptide hydrogels modulate in vitro chondrogenesis of bovine bone marrow stromal cells. Tissue Eng Part A 16, 465, 2010.

13. Florine, E.M., Miller, R.E., Porter, R.M., Evans, C.H., Kurz, B., and Grodzinsky, A.J. Effects of dexamethasone on mesenchymal stromal cell chondrogenesis and aggrecanase activity: comparison of agarose and self-assembling peptide scaffolds. Cartilage 4, 63, 2013.

14. Kopesky, P.W., Vanderploeg, E.J., Kisiday, J.D., Frisbie, D.D., Sandy, J.D., and Grodzinsky, A.J. Controlled delivery of transforming growth factor betal by self-assembling peptide hydrogels induces chondrogenesis of bone marrow stromal cells and modulates Smad2/3 signaling. Tissue Eng Part A 17, 83, 2011.

15. Dickhut, A., Gottwald, E., Steck, E., Heisel, C., and Richter, W. Chondrogenesis of mesenchymal stem cells in gel-like biomaterials in vitro and in vivo. Front Biosci 13, 4517, 2008.

16. Ley, K., Cerrito, M., and Arfors, K.E. Sulfated polysaccharides inhibit leukocyte rolling in rabbit mesentery venules. Am J Physiol 260, H1667, 1991.

17. Bonassar, L.J., Grodzinsky, A.J., Srinivasan, A., Davila, S.G., and Trippel, S.B. Mechanical and physicochemical regulation of the action of insulin-like growth factor-I on articular cartilage. Arch Biochem Biophys 379, 57, 2000.

18. Ragan, P.M., Chin, V.I., Hung, H.H., Masuda, K., Thonar, E.J., Arner, E.C., Grodzinsky, A.J., and Sandy, J.D. Chondrocyte extracellular matrix synthesis and turnover are influenced by static compression in a new alginate disk culture system. Arch Biochem Biophys 383, 256, 2000.

19. Trippel, S.B., Corvol, M.T., Dumontier, M.F., Rappaport, R., Hung, H.H., and Mankin, H.J. Effect of somatomedinC/insulin-like growth factor I and growth hormone on cultured growth plate and articular chondrocytes. Pediatr Res 25, 76, 1989.

20. Sah, R.L., Kim, Y.J., Doong, J.Y., Grodzinsky, A.J., Plaas, A.H., and Sandy, J.D. Biosynthetic response of cartilage explants to dynamic compression. J Orthop Res 7, 619, 1989.

21. Kim, Y.J., Sah, R.L., Doong, J.Y., and Grodzinsky, A.J. Fluorometric assay of DNA in cartilage explants using Hoechst 33258. Anal Biochem 174, 168, 1988.

22. Yang, Y., Khoe, U., Wang, X., Horii, A., Yokoi, H., and Zhang, S. Designer self-assembling peptide nanomaterials. Nano Today 4, 193, 2009.

23. Farndale, R.W., Sayers, C.A., and Barrett, A.J. A direct spectrophotometric microassay for sulfated glycosaminoglycans in cartilage cultures. Connect Tissue Res 9, 247, 1982.

24. Hsieh, P.C., Davis, M.E., Gannon, J., MacGillivray, C., and Lee, R.T. Controlled delivery of PDGF-BB for myocardial protection using injectable self-assembling peptide nanofibers. J Clin Invest 116, 237, 2006. 
25. Stegemann, H., and Stalder, K. Determination of hydroxyproline. Clin Chim Acta 18, 267, 1967.

26. Sato, A., Nishimura, S., Ohkubo, T., Kyogoku, Y., Koyama, S., Kobayashi, M., Yasuda, T., and Kobayashi, Y. Threedimensional structure of human insulin-like growth factor-I (IGF-I) determined by $1 \mathrm{H}-\mathrm{NMR}$ and distance geometry. Int J Pept Protein Res 41, 433, 1993.

27. Morales, T.I. The insulin-like growth factor binding proteins in uncultured human cartilage: increases in insulinlike growth factor binding protein 3 during osteoarthritis. Arthritis Rheum 46, 2358, 2002.

28. Erickson, I.E., van Veen, S.C., Sengupta, S., Kestle, S.R., and Mauck, R.L. Cartilage matrix formation by bovine mesenchymal stem cells in three-dimensional culture is age-dependent. Clin Orthop Relat Res 469, 2744, 2011.

29. Kopesky, P.W., Lee, H.Y., Vanderploeg, E.J., Kisiday, J.D., Frisbie, D.D., Plaas, A.H., Ortiz, C., and Grodzinsky, A.J. Adult equine bone marrow stromal cells produce a cartilage-like ECM mechanically superior to animal-matched adult chondrocytes. Matrix Biol 29, 427, 2010 .
30. De Bari, C., Kurth, T.B., and Augello, A. Mesenchymal stem cells from development to postnatal joint homeostasis, aging, and disease. Birth Defects Res C Embryo Today 90, $257,2010$.

31. Tran-Khanh, N., Hoemann, C.D., McKee, M.D., Henderson, J.E., and Buschmann, M.D. Aged bovine chondrocytes display a diminished capacity to produce a collagen-rich, mechanically functional cartilage extracellular matrix. J Orthop Res 23, 1354, 2005.

Address correspondence to: Alan J. Grodzinsky, ScD MIT Room NE47-377 500 Technology Square Cambridge, MA 02139

E-mail: alg@mit.edu

Received: November 3, 2013 Accepted: September 15, 2014 Online Publication Date: October 21, 2014 